Ana Cristina Brito 1

Márcio Caval canteVila-Nova 1

Deisy Alécia Martins Rocha 1

Lidiana Gomes Costa 1

Wendell Alexandre Pinheiro de Almeida 1

Luciana da Silva Viana 1

Ricardo Ramalho Lopes J. 1

Gil berto Fontes 1

Eliana Maria Maurício da Rocha 1

Lêda Regis 2

\section{Prevalência da filariose canina causada por Dirofilaria immitis e Dipetalonema reconditum em Maceió, Alagoas, Brasil}

\author{
Prevalence of canine filariasis by Dirofilaria \\ immitis and Dipetalonema reconditum \\ in Maceió, Alagoas State, Brazil
}

1 Departamento de Patologia, Centro de Ciências Biológicas, Universidade Federal de Alagoas. Praça Afrânio Jorge s/n, Maceió, AL 57010-020, Brasil. acbs@fapeal.br 2 Departamento de Entomologia, Centro de Pesquisas Aggeu Magal hães, Fundação Oswaldo Cruz. Campus da Universidade Federal de Pernambuco, Av. Moraes Rego s/n, Recife, PE 50670-420, Brasil.
Abstract A survey on the prevalence of Dirofilaria immitis and Dipetalonema reconditum was conducted in 1,519 dogs from Maceió and two coastal areas in the State of Alagoas, Northeast Brazil, from 1995 to 1999, by testing for microfilariae in blood. All blood samples were from exclusi vely domiciled dogs with a known history, showing that the infections were autochthonous, confirming transmission of canine filariasis in these areas. In Greater Metropolitan Maceió, 15 (1.3\%) microfilaremic dogs were detected with D. immitis and 15 (1,3\%) with D. reconditum. In the southern coastal area there was an estimated prevalence of $12.7 \%$ for D. immitis. D. immitis and D. reconditum microfilaria were $298.1 \mu \mathrm{m}$ and $249.2 \mu \mathrm{m}$ long and $7.3 \mu \mathrm{m}$ and $4.4 \mu \mathrm{m}$ wide, respectively. A Witness immunotest that detects $D$. immitis antigen was used to confirm parasitological results and reveal occult dirofilariasis cases. Of the total 6,579 femal es examined, 8 (0.1\%) Culex quinquefasciatus were observed to be naturally infected with $\mathrm{D}$. immitis larvae. These results proved dirofilariasis transmission in Maceió and demonstrated D. reconditum in the same geographic area.

Key words Dirofilariasis; Dirofilaria immitis; Dipetalonema reconditum

Resumo A prevalência da filariose canina causada por Dirofilaria immitis e Dipetalonema reconditum, foi estimada após a realização de inquérito hemoscópico em 1.519 cães domiciliados. Foram exami nados 1.097 cães nos sete distritos sanitários da cidade de Maceió, Alagoas, 204 na Il ha de Santa Rita e 218 na cidade de Paripueira. Em Maceió, foram detectados 15 (1,3\%) cães com microfilárias (MF) de D. immitis e 15 (1,3\%) com MF de D. reconditum. Todos os casos posi tivos foram considerados autóctones. As MF de D. immitis e de D. reconditum mediram 298,1 $\mu \mathrm{m}$

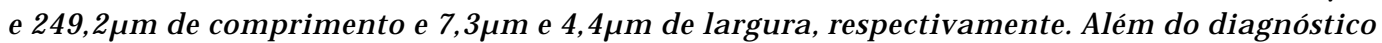
parasi tológi co pela técnica de gota espessa $(100 \mu \mathrm{L})$, foi utilizado o teste imunológico Witness, para detecção de antígeno filarial circulante, o qual confirmou os dados parasitológicos e permitiu a detecção de casos de infecção oculta. De um total de 6.579 fêmeas examinadas, pertencentes a sei s espéci es de mosquitos, oi to $(0,1 \%)$ fêmeas de Culex quinquefasciatus foram encontradas naturalmente infectadas com larvas de D. immitis. Estes resultados demonstram a presença simultânea de D. immitis e D. reconditum na mesma área geográfica.

Palavras-chave Dirofilariose; Dirofilaria immitis; Dipetalonema reconditum 
Introdução

Dirofilaria immitis e Dipetal onema reconditum, helmintos causadores da filariose em cães e gatos, apresentam ocorrência simultânea em várias regiões geográficas e produzem microfilárias (MF) que circulam no sangue dos hospedeiros naturais (Patton \& Faulkner, 1992).

A diferenciação entre essas espécies é importante, uma vez que a infecção por D. i immitis em cães, pode resultar em doença e morte, enquanto que a infecção por D. reconditum é transitória e sem conseqüências patológicas (Knight, 1987). Além disso, no caso de D. immitis, há riscos de transmissão ao homem, tendo como resultado a dirofilariose pulmonar humana, sendo considerada portanto, zoonose (Rodrigues-Silva et al., 1995).

O diagnóstico da filariose tem sido geralmente baseado no encontro de MF no sangue. As técnicas de detecção de M F incluem gotas espessas (Knigth, 1977), testes de concentração como a técnica de Knott (Newton \& Wright, 1956) e filtração em membrana de policarbonato (Chularerk \& Desowitz, 1970). Técnicas de imunodiagnóstico foram desenvolvidas com o objetivo de detectar as formas ocultas da infecção. Recentemente, o uso da técnica molecular utilizando a reação em cadeia da polimerase (PCR), foi proposto como método espécie-específico de diagnóstico da dirofilariose canina, por Nicolas \& Scoles (1997).

No Brasil, os estudos revelam que as mais altas prevalências de filariose canina foram encontradas nos estados do Maranhão (46\%), São Paulo (14,2\%), Rio de Janeiro (13,68\%) e Minas Gerais (9,4\%) (Ahid et al., 1999; Duque-Araújo et al., 1995; Ferreira et al., 1999; Labarthe et al., 1998). No entanto, considerando as diferentes características climáticas e de extensão territorial, a prevalência de dirofilariose canina no Brasil, e particularmente, no Nordeste, é pouco conhecida.

Na cidade de Maceió, Alagoas, a prevalência de filariose canina foi estimada, em estudo preliminar, em 3,1\% (Brito et al., 2000). No entanto, a real prevalência da infecção filarial causada por D. immitis e por D. reconditum nesta área permanece desconhecida.

O presente estudo apresenta os dados da prevalência desses filarídeos estimada a partir de estudo por busca ativa, em amostra representativa de cães domiciliados nos sete distritos sanitários da cidade de Maceió e em duas áreas litorâneas, localizadas ao norte e ao sul da cidade, bem como a identificação de vetores naturais de $D$. immitis.
Metodologia

Descrição das áreas

Maceió, capital do Estado de Alagoas, está situada no litoral médio do Estado entre os meridianos 350 44' W e 350 56' W e os paralelos 9o 35' Se 9024 ' S. A população urbana é de 630.000 habitantes distribuídos em área de $208 \mathrm{~km}^{2}$ (IBGE, 1991). A temperatura média mensal varia de $24 \circ \mathrm{C}$ a $28 \circ \mathrm{C}$ e a umidade de $60 \%$ a $80 \%$. A área urbana é dividida em sete distritos sanitários, sendo que $80 \%$ da população dispõe de água tratada e somente $27 \%$ das habitações são servidas por rede de esgoto básico (IMPAR, 1995).

Duas outras regiões foram selecionadas por serem as primeiras áreas banhadas por águas oceânicas ou lagunares, fora do Município de Maceió: a Ilha de Santa Rita, litoral sul, maior ilha lacustre do Brasil (Ramalho et al., 1985), com população estimada de cinco mil habitantes e a cidade de Paripueira, área urbana localizada ao litoral norte, distante cerca de $25 \mathrm{~km}$ do centro de Maceió, com população de 4.500 habitantes.

\section{A população alvo}

A população canina incluída nesta investigação era formada estritamente por cães domiciliados, de ambos os sexos, com idade igual ou maior a nove meses, sem raça definida ou de variadas raças.

\section{Amostra}

O inquérito hemoscópico canino foi realizado nos sete distritos sanitários da cidade de Maceió, de janeiro de 1997 a dezembro de 1999. O tamanho da amostra foi calculado juntamente com a equipe de estatística do Centro de Pesquisas Aggeu Magalhães, Fundação Oswaldo Cruz (FIOCRUZ), Pernambuco, tomando como base a prevalência preliminarmente estimada em 3,1\% para filariose canina na cidade de Maceió (Brito et al., 2000), precisão absoluta de $2 \%$ e o critério utilizado pela Organização Mundial de Saúde, de que a população de cães pode ser estimada em $10 \%$ da população humana (WHO Expert Committee on Rabies, 1994). Foram examinados 1.097 cães na área urbana de Maceió, sendo 626 machos e 471 fêmeas. Os bairros e as ruas onde o inquérito hemoscópico foi realizado foram sorteados, aleatoriamente, assim como também a primeira casa de cada rua eleita. A partir deste ponto, a coleta das amostras seguiu o sentido horário, até que o número determinado fosse alcançado. 
Nas duas outras regiões, por serem áreas menores, foram avaliados todos os cães domiciliados encontrados, considerando o pequeno universo da população canina. Assim, foram examinados 204 cães na Ilha de Santa Rita, sendo 139 machos e 65 fêmeas e 218 animais (104 machos e 114 fêmeas) na cidade de Paripueira. Informações sobre idade, sexo, raça, local de nascimento e tempo de residência na área foram obtidas para cada cão, visando verificar a autoctonia dos casos. Nenhum dos cães investigados tinha sido previamente examinado ou tratado para filariose canina.

Método de coleta

A técnica utilizada para a pesquisa de microfilárias circulantes foi a de gota espessa de sangue do lobo distal da orelha de cada animal, no horário entre 9 e 17 horas. Foram preparadas

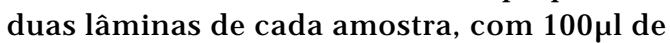
sangue cada. Após a coleta, as lâminas foram processadas e coradas com Giemsa no laboratório do Departamento de Patologia do Centro de Ciências Biológicas da Universidade Federal de Alagoas. O exame ao microscópio óptico foi feito com aumento de 100X, com revisão de $100 \%$ das amostras.

Identificação da espécie de filarídeo nos cães infectados

Amostras de $5 \mathrm{ml}$ de sangue foram retiradas da veia radial da pata traseira de todos os animais microfilarêmicos, transferidas para tubos contendo anticoagulante (Heparina) e submetidas a duas técnicas de diagnóstico parasitológico e ao teste imunológico Witness Dirofilaria (Merial, Campinas, Brasil). O exame a fresco entre lâmina e lamínula e a técnica de Knott modificada, foram realizados com o objetivo de obter identificação específica baseada na motilidade e tamanho das MF, bem como na morfologia das regiões anterior e posterior destas. As medidas de comprimento e largura foram obtidas de quinze MF de cada lâmina de cão infectado, com auxílio de ocular micrométrica.

O teste imunológico Witness, apresenta-se em forma de cartão para diagnóstico individual baseado na tecnologia de imunomigração rápida, usando anticorpos dirigidos contra epítopos específicos de antígeno de superfície de verme adulto de D. immitis. Este teste foi utilizado em 239 dos 1.097 animais examinados, a fim de verificar a ocorrência de dirofilariose oculta. Além disso, foram necropsiados seis cães infectados, com o objetivo de avaliar a freqüência de vermes adultos e de confirmar a etiologia das infecções locais. O coração e os pulmões foram examinados individualmente, a fim de registrar o número e o sexo dos helmintos encontrados.

\section{Índices de infecção vetorial natural}

Com o objetivo de identificar os vetores da filariose canina, foram realizadas coletas de mosquitos durante 24 meses (janeiro/ 1998 a dezembro/1999), duas a três vezes por semana, no intradomicílio onde residiam os cães infectados. Na área urbana de Maceió, as coletas foram realizadas com capturador manual duas vezes ao dia: pela manhã, entre 8 e 9 horas eà tarde, das 16 às 17 horas. Na Ilha de Santa Rita, além da captura manual, foram usadas armadilhas luminosas do tipo CDC miniatura, com luz branca (incandescente) e negra (UV), durante o dia e à noite. Após a coleta, $50 \%$ dos mosquitos eram separados, colocados a $-20 \circ \mathrm{C}$ e identificados. Posteriormente eram dissecados separando-se cabeça, tórax, abdômen e túbulos de Mal pighi, para exame ao microscópio. Os exemplares vivos eram mantidos no insetário durante 15 dias e então dissecados de forma semelhante aos anteriores.

\section{Análise estatística}

O teste qui-quadrado foi utilizado para análise de significância estatística das diferenças encontradas na prevalência da infecção nos diferentes distritos avaliados, bem como para comparação de freqüência da dirofilariose entre os sexos dos animais infectados. O programa utilizado foi o Epi Info versão 6.0 (CDC/ WHO, 1996).

\section{Resultados}

A distinção entre MF das duas espécies foi feita com base em critérios de tamanho e movimento, segundo Freitas (1976). No exame a fresco, as diferenças quanto ao movimento são bastante claras: as M F de D. immitis realizam movimentos ondulantes do tipo serpentiforme, deslocando-se lentamente no campo, enquanto as de $\mathrm{D}$. reconditum deslocam-se com maior rapidez, movendo-se erraticamente. As MF de D. immitis são maiores, medindo $298,1 \pm 5,5 \mu \mathrm{m}$ de comprimento e 7,3 $\pm 0,3 \mu \mathrm{m}$ de largura, enquanto que as $M F$ de $D$. reconditum apresentam $249,2 \pm 8,7 \mu \mathrm{m}$ de comprimento e 4,4 \pm $1,2 \mu \mathrm{m}$ de largura, valores concordantes com os observados por Lindsey (1965) e Falls \& Platt (1982). No presente estudo foi observado que a 
extremidade anterior das MF de D. immitis é ovalada, enquanto que em $D$. reconditum é achatada. A extremidade posterior da primeira espécie é reta e a da segunda, em forma de gancho.

Dos 858 cães examinados utilizando apenas gota espessa, 486 eram machos e 372 fêmeas. Do total, 18 (2,1\%) (IC 95\%: 1,25-3,30) animais apresentaram-se microfilarêmicos, sendo 13 machos e cinco fêmeas. Após a realização do teste de Knott, sete $(0,8 \%)$ cães estavam infectados apenas com $D$. immitis e 11 (1,3\%) com D. reconditum.

Em um segundo grupo formado por 239 cães, cujas amostras sangüíneas foram examinadas, simultaneamente, pela gota espessa e pelo Witness, foram encontrados $12(5,0 \%)$ cães infectados. Destes, nove foram detectados pela técnica de gota espessa e, após avaliação pelo teste de Knott, três (1,2\%) estavam infectados com D. immitis, quatro $(1,6 \%)$ com $D$. reconditum e dois $(0,8 \%)$ com infecção mista de ambas as espécies. $\mathrm{O}$ teste Witness detectou todos os cães que apresentaram MF de D. immitis diagnosticados por gota espessa e mais três animais cuja microfilaremia não foi detectada pelos exames parasitológicos de gota espessa e de Knott. Estes animais foram considerados portadores de uma possível infecção oculta.

No total, dos 1.097 cães examinados nos sete distritos sanitários da área urbana de Maceió, trinta $(2,73 \%)$ (IC 95\%: 1,85-3,88) apresentaram-se infectados, sendo 15 (1,3\%) com D. immitis e 15 (1,3\%) com D. reconditum. No grupo de cães infectados por D. immitis, dois cães apresentaram infecção mista por ambas as espécies. A prevalência entre cães machos foi de 3,19\% (IC 95\%: 1,96-4,89) e entre as fêmeas de 2,12\% (IC 95\%: 1,02-3,87), não sendo esta diferença estatisticamente significativa $\left(\chi^{2}=1,16 ; p=0,28\right)$. Os distritos 1,2 e 4, localizados no litoral, apresentaram prevalência de filariose canina de 3,70\% (IC 95\%: 2,4-5,4), enquanto que naqueles localizados no centro da cidade, a prevalência estimada foi de 1,2\% (IC 95\%: 0,4-2,7). Esta diferença foi estatisticamente significativa $\left(\chi^{2}=6,19 ; p=0,013\right)$. Analisando os casos positivos para D. immitis, foi estimada a prevalência de 1,78\% (IC 95\%: 0,923,08 ) nos distritos do litoral e de 0,71\% (IC 95\%: $0,15-2,06)$ nos demais, não sendo esta diferença significativa $\left(\chi^{2}=2,19 ; p=0,14\right)$ (Tabela 1 , Figura 1).

Não foram observados cães infectados com filarídeos na área do litoral norte, entre os 218 examinados. Dos 204 cães examinados na Ilha de Santa Rita, litoral sul, 26 (12,7\%) (IC 95\%: 8,49-18,11) apresentaram MF de D. immitis.
Foram recuperados 72 vermes adultos ( $37 \mathrm{ma}$ chos e 35 fêmeas) nos seis cães necropsiados, variando entre 2 a 49 vermes por animal infectado. Em apenas um cão foi observada a presença de verme adulto no pulmão.

Os dados referentes às espécies de mosquitos capturados nas áreas investigadas neste estudo, estão demonstrados na Tabela 2. Nas coletas realizadas na área urbana de Maceió, apenas exemplares das espécies Cul ex quinquefasciatus e Aedes aegypti foram coletados. Na Il ha de Santa Rita, seis espécies, pertencentes a três gêneros, foram identificadas: $C x$. quinquefasciatus, Ae. taeni orhynchus, Ae. aegypti, Ae. scapularis, Ae. albifasciatuse Anopheles darlingi . Cx. quinquefasciatus foi predominante (93\%) entre as espécies capturadas manualmente ou com armadilhas luminosas, tanto na área urbana de Maceió, quanto na Ilha de Santa Rita. Nesta área, a segunda espécie mais freqüente foi o Ae. taeniorhynchus (3,3\%), seguida de Ae. scapularis. Não foi realizada coleta de vetor na cidade de Paripueira, uma vez que não foram detectados cães infectados.

Apenas oito Cx. quinquefasciatus das 6.579 fêmeas examinadas, foram encontradas albergando larvas de D. immitis nos túbulos de Malpighi, sendo cinco exemplares oriundos do segundo distrito da área urbana de Maceió e três da Il ha de Santa Rita (índice de infecção vetorial $=0,1 \%$ ).

\section{Discussão}

Todos os casos de filariose canina detectados neste estudo foram considerados autóctones, uma vez que os animais infectados nasceram nas áreas da investigação e não foram reportados deslocamentos para outras regiões endêmicas de dirofilariose.

Na presente investigação não foi observada diferença significativa na prevalência da infecção entre os sexos dos animais. Este resultado está de acordo com as observações de Falls \& Platt (1982) e Ahid et al.(1999). No entanto, relatos na literatura demonstraram prevalência duas a quatro vezes maior para os cães machos do que para fêmeas (Alves et al., 1999; Wallenstein \& Tibola, 1960). Alguns fatores como estado fisiológico do animal, idade e exposição aos vetores, podem estar relacionados com a prevalência menor da infecção entre as fêmeas (Perez-Sanchez et al., 1989).

As prevalências de filariose canina em Maceió, foram estatisticamente maiores em áreas banhadas por águas oceânicas e/ ou lagunares ou próximas destas, como se pode observar na 
Prevalência da filariose canina causada por Dirofilaria immitis e por Dipetalonema reconditum. Maceió, Alagoas.

\begin{tabular}{|c|c|c|c|c|c|c|c|}
\hline \multirow[t]{2}{*}{ Distritos de Maceió } & \multirow[t]{2}{*}{ Cães examinados } & \multicolumn{2}{|c|}{ Cães infectados } & \multicolumn{2}{|c|}{$\begin{array}{l}\text { Infecção por } \\
\text { D. immits }\end{array}$} & \multicolumn{2}{|c|}{$\begin{array}{l}\text { Infecção por } \\
\text { D. reconditum }\end{array}$} \\
\hline & & $n$ & $\%$ & $\mathrm{n}$ & $\%$ & $\mathrm{n}$ & $\%$ \\
\hline Litoral (1, 2 e 4$)$ & 675 & 25 & 3,70 & 12 & $1,78 *$ & 13 & 1,92 \\
\hline Centro $(3,5,6$ e 7$)$ & 422 & 5 & 1,18 & 3 & 0,71 & 2 & 0,47 \\
\hline Total & 1.097 & 30 & 2,72 & 15 & 1,36 & 15 & 1,36 \\
\hline
\end{tabular}

* Incluindo dois casos de infecção mista, com ambas as espécies.

Figura 1. Estas áreas são mais úmidas e apresentam maior área verde, o que certamente contribui para a formação de criadouros de culicídeos.

Estes dados confirmam as investigações de Ahid et al. (1999) e Labarthe et al. (1998) que demonstraram os maiores índices de dirofilariose nos cães que procederam da orla marítima ou de áreas próximas a estas. Estes resultados permitem supor que a infecção tenha se estabel ecido originalmente na faixa litorânea de Maceió, e que os focos de infecção distantes do litoral tenham se formado pela migração de cães microfilarêmicos para aquelas áreas. Com efeito, no sexto distrito mais distante do litoral, onde foram registrados casos positivos para $D$. immitis, todos os cães infectados haviam migrado do primeiro distrito. Em relação aos cães infectados por D. reconditum, foi observado que a maioria vive em áreas cujas condições sócio-econômicas e sanitárias são mais precárias, o que facilitaria a proliferação de pulgas e conseqüentemente a transmissão do filarídeo.

Estudos recentes realizados em diferentes áreas endêmicas para D. immitis no Brasil, revelaram altas prevalências de microfilaremia ( $9 \%$ a $46 \%$ ) em Minas Gerais, Rio de Janeiro, São Paulo e Maranhão (Ahid et al., 1999; Duque-Araújo et al., 1995; Ferreira et al., 1999; Labarthe et al., 1998). Em Maceió, uma baixa prevalência foi registrada no presente estudo, semelhante à estimada por Alves et al. (1999) para Recife, Pernambuco, cidade situada cerca de $250 \mathrm{~km}$ de Maceió. Estes dados sugerem que a dirofilariose canina pode estar ainda em processo de expansão nestas duas áreas do Nordeste. Por outro lado, uma alta prevalência de microfilaremia foi observada em área litorânea muito próxima de Maceió, a ilha lacustre de Santa Rita, sugerindo que a transmissão de D. immitis se estabeleceu há mais tempo ali do que na área urbana de Maceió. As condições ambientais naquela ilha, com vegetação litorâ-
Figura 1

Distritos Sanitários da Cidade de Maceió: 1, 2 e 4 (Litoral); 3, 5, 6 e 7 (Centro).

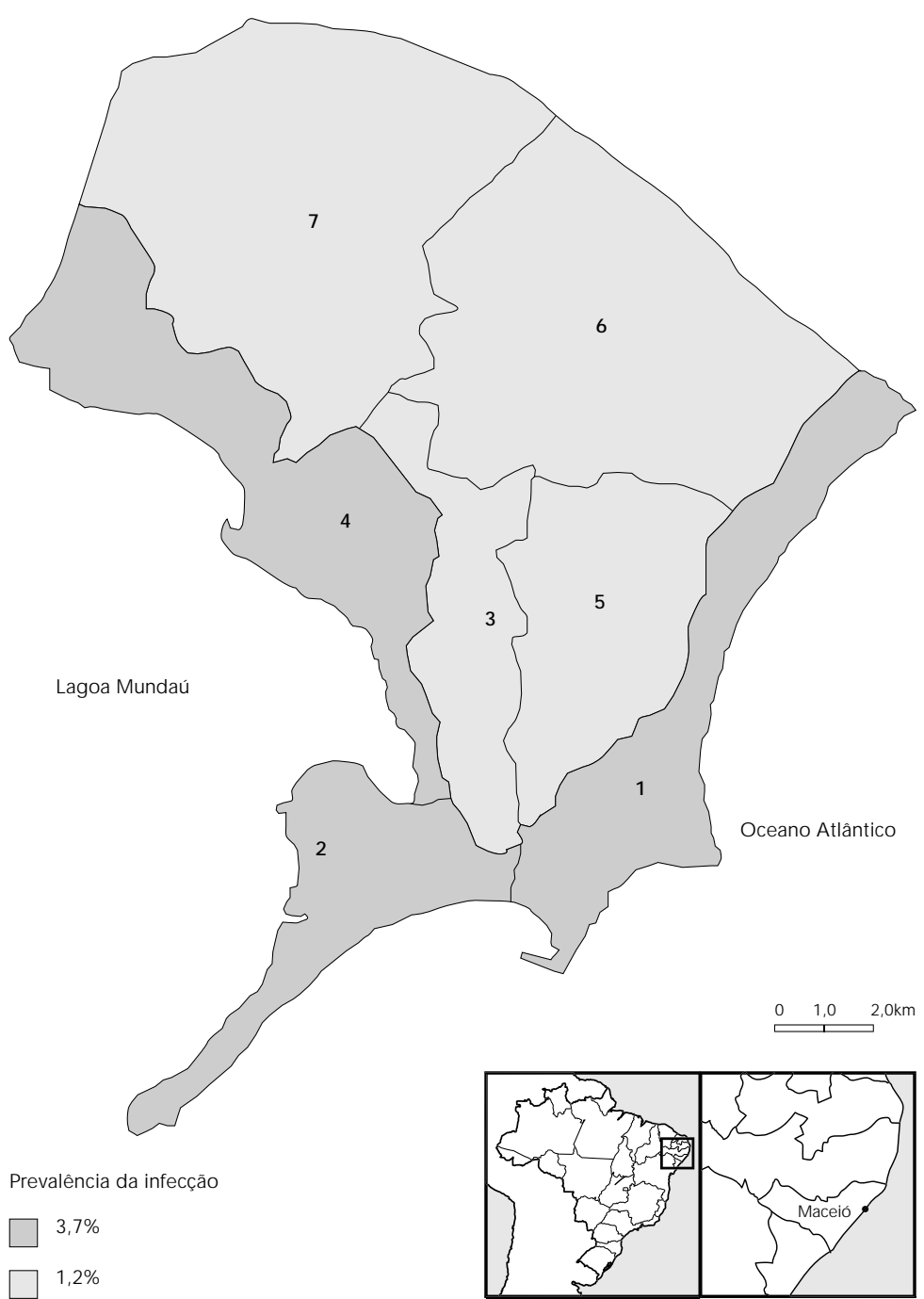


Freqüência de fêmeas capturadas em Maceió e na Ilha de Santa Rita, com capturador de Castro (manual) e armadilha luminosa. J aneiro de 1998 a dezembro de 1999.

\begin{tabular}{|c|c|c|c|c|c|c|}
\hline \multirow[t]{2}{*}{ Espécies } & \multicolumn{2}{|c|}{ Armadilha } & \multicolumn{2}{|c|}{ Manual } & \multicolumn{2}{|c|}{ Total } \\
\hline & Freqüência & $\%$ & Freqüência & $\%$ & Freqüência & $\%$ \\
\hline Culex quinquefasciatus & 2.878 & 46,0 & 3.336 & 54,0 & 6.214 & 93,0 \\
\hline Aedes taeniorhynchus* & 126 & 58,0 & 92 & 42,0 & 218 & 3,3 \\
\hline Aedes aegypti & 2 & 3,0 & 80 & 97,0 & 82 & 1,2 \\
\hline Aedes scapularis* & 15 & 26,0 & 43 & 74,0 & 58 & 0,8 \\
\hline Anopheles darlingi* & 5 & 84,0 & 1 & 16,0 & 6 & 0,09 \\
\hline Aedes albisfasciatus* & 0 & 0,0 & 1 & 100,0 & 1 & 0,01 \\
\hline Total & 3.026 & 46,0 & 3.553 & 54,0 & 6.579 & 100,0 \\
\hline
\end{tabular}

* Espécies capturadas apenas na Ilha de Santa Rita.

nea diversificada e exuberante e a presença de muitas coleções de águas, são altamente favoráveis ao desenvolvimento de mosquitos, responsáveis pela transmissão da infecção (RamaIho et al., 1985).

A cidade de Paripueira, onde não foram encontrados cães infectados, é uma área com as características geográficas já bastante modificadas pela ação do homem. Acredita-se que a existência de coleta regular de lixo, ruas calçadas, programas de combate ao vetor do dengue com inseticidas e progressivo desmatamento das áreas florestais para construções de obras civis, podem ser alguns dos fatores limitantes da proliferação do vetor e estabelecimento da infecção.

Um provável quadro de dirofilariose oculta foi demonstrado em três dos seis, ou seja, em $50 \%$ dos cães infectados por D. immitis na amostra avaliada pelo Witness, confirmando os dados da literatura de que cerca de $10 \%$ a $67 \%$ dos cães infectados são portadores de infecção oculta (Otto, 1978).

Algumas evidências indiretas foram consideradas no sentido de inferir que as $L_{1}$ encontradas nos túbulos de Malpighi dos Cx. quinquefasciatus eram de D. immitis. Inicialmente, foi comprovado que não havia na habitação, cães infectados por D. reconditum e que os mosquitos positivos foram capturados próximos ao local de repouso de cães com alta densidade de microfilárias de $\mathrm{D}$. immitis. Além disso, a pesquisa para Wuchereria bancrofti, outro filarídeo presente em algumas áreas da cidade (Fontes et al., 1994) foi realizada por gota espessa, em todos os indivíduos moradores do domicílio e todos foram negativos. Embora não tenham sido encontradas larvas infectantes (L3) nas fêmeas infectadas, mas apenas L1, acredita-se que o Cx. quinquefasciatus está envolvido na transmissão da dirofilariose nesta área, uma vez que todos os exemplares infectados foram capturados na região litorânea de maior prevalência da infecção e esta espécie já foi identificada anteriormente como transmissora do parasito em outras regiões do Brasil (Ahid \& Lourenço-de-Oliveira, 1999; Labarthe et al., 1998), bem como em outros países (Ludlam et al., 1970; Parker, 1993; Sauerman \& Nayar, 1983; Villavaso \& Steelman, 1970).

Os dados obtidos no presente estudo, confirmam as observações de Labarthe et al. (1992), de que em áreas onde a culicidofauna é representada, predominantemente, por espécie com baixa competência vetorial, como o $\mathrm{Cx}$. quinquefasciatus, a freqüência de dirofilariose canina é baixa.

Ae taeniorhynchus é comprovadamente vetor importante de D. immitis em Itacoatiara, Niterói, Rio de Janeiro (Labarthe et al., 1998), em São Luís, Maranhão (Ahid \& Lourenço-deOliveira, 1999), além de ser o vetor primário na Flórida e Carolina do Norte, Estados Unidos (Parker, 1986, 1993; Sauerman \& Nayar, 1983). Ae. scapularis, outro culicídeo encontrado na área de estudo, é considerado vetor primário de D. immitis no Rio de Janeiro (Labarthe et al., 1998). Diante desses dados, não se pode descartar a possibilidade das mesmas estarem envolvidas na transmissão de D. immitis em Maceió, mesmo considerando que não foram encontradas fêmeas dessas duas espécies infectadas com o parasito nesta área. É necessário esclarecer que o número de fêmeas dessas espécies capturadas e examinadas neste estudo, não foi significativo, devido à baixa densidade populacional das mesmas na área. Novos estudos são portanto necessários para avaliar a im- 
portância local destas espécies na transmissão da dirofilariose.

A presente investigação demonstrou a ocorrência simultânea de $D$. immitise $D$. reconditum na cidade de Maceió, Alagoas. De acordo com as observações de Muro et al. (1999), a presença da filariose canina causada por D. immitis em uma determinada região, sugere que a dirofilariose pulmonar humana deve ser considerada no diagnóstico diferencial de neopla- sias e infecções fúngicas pulmonares. Campos et al . (1997) reportaram a ocorrência de 27 casos de dirofilariose pulmonar humana em São Paulo. No entanto, na Região Nordeste, não há relatos publicados sobre o tema. Estudos sobre a ocorrência desta infecção em humanos devem ser realizados, a fim de investigar o caráter zoonótico da dirofilariose canina nas áreas da presente investigação.

\section{Referências}

AHID, S. M. M. \& LOURENÇO-DE-OLIVEIRA, R., 1999. Mosquitos vetores potenciais de dirofilariose canina na região nordeste do Brasil. Revista deSaúde Pública, 33:560-565.

AHID, S. M. M.; LOURENÇO-DE-OLIVEIRA, R. \& SARAIVA, L. Q., 1999. Dirofilariose canina na ilha de São Luís, Nordeste do Brasil: Uma zoonose potencial. Cadernos deSaúdePública, 15:405-412.

ALVES, L. C.; SILVA, L. V. A.; FAUSTINO, M. A. G.; McCALL, J. W.; SUPAKONDERJ, P.; LABARTHE, N. W.; SANCHEZ, M. \& CAIRES, O., 1999. Survey of canine heartworm in the city of Recife, Pernambuco, Brazil. Memórias do Instituto Oswaldo Cruz, 94:587-590.

BRITO, A. C.; VIANA, L. S.; DUARTE, E. M.; ROCHA, E. M. M.; FONTES, G. \& REGIS, L., 2000. Dirofilaria immitis infection in dogs from Maceió-Alagoas, Northeast region of Brazil. Arquivo de Medicina Veterinária eZootecnia, 52:210-211.

CAMPOS, J. R. M.; BARBAS, C. S. V.; FILOMENO, L. T. B.; FERNANDEZ, A.; MINAMOTO, H.; BARBAS FILHO, J. V. \& JATENE, F. B., 1997. Human pulmonary dirofilariasis. Chest, 112:729-733.

CDC (Centers for Disease Control and Prevention)/ WHO (World Health Organization), 1996. Epi Info 6, Version 6.04. A Word Processing, Database, and Statistics Programs for Public Health. Atlanta: CDC/Geneva: WHO.

CHULARERK, P. \& DESOWITZ, R. S., 1970. A simplified membrane filtracion technique for the diagnosis of microfilaraemia. Journal of Parasitology, 53:623-624.

DUQUE-ARAÚJO, A. M.; LABARTHE, N.; LUVISÁRIO, S. L. \& REINA, D., 1995. Filariose canina no Estado de São Paulo - Brasil. In: IV Congresso Ibérico de Parasitologia, Anais, pp. 93-94. Santiago de Compostela: Sociedade I bérica de Parasitologia.
FALLS, R. K. \& PLATT, T. R., 1982. Survey for heartworm, Dirofilaria immitis and Dipetalonema re conditum (Nematoda: Filarioidea) in dogs from Virginia and North Carolina. American Journal of Veterinary Research, 43:738-739.

FERREIRA, F. A.; BARBOSA, F. C.; MASTRANTONIO, E. C. \& FARACIO, A. D., 1999. Ocorrência da dirofilariose canina na cidade de Uberlândia, MG, Brasil. Veterinária Notícias, Uberlândia, 5:57-61.

FONTES, G.; BRITO, A. C.; CALHEIROS, C. M. L. \& ROCHA, E. M. M., 1994. Situação atual da filariose bancroftiana na cidade de Maceió, Estado de Alagoas, Brasil. Cadernos de Saúde Pública, 10:293-300.

FREITAS, M. G., 1976. Helmintologia Veterinária. Belo Horizonte: Editora Rabelo \& Brasil.

IBGE (Fundação Instituto Brasileiro de Geografia e Estatística), 1991. X Recenseamento Geral Brasilei ro (Alagoas, Censo Demográfico, Dados Distritais), n. 15. Maceió: IBGE.

IMPAR (Instituto Municipal de Planejamento e Ação Regional) 1995. Dados sobre População da Cidade de Maceió. Maceió: Prefeitura Municipal de Maceió.

KNIGHT, D. H., 1977. Heartworm heart disease. Advances of Veterinary Sciences and Comparative Medicine, 21:107-149.

KNIGHT, D. H., 1987. Heartworm infection. Veterinary Clinics of North America. Small Animals Practice, 17:1463-1518.

LABARTHE, N. V.; ARAÚJO, A. M.; BORDIN, E. L.; LARSSON, M. E. \& GUERRERO, J., 1992. Update on the distribution of Dirofilaria immitis in dogs in Brazil. In: XVII WSAVA World Congress, Proceedings, pp. 287-289. Rome: World Small Animal Veterinary Association.

LABARTHE, N.; SERRÃO, M. L.; MELO, Y. F.; OLIVEIRA, S. J. \& LOURENÇO-DE-OLIVEIRA, R., 1998. 
Potential vectors of Dirofilaria immitis (Leidy, 1856) in Itacoatiara, Oceanic Region of Niterói Municipality, State of Rio de Janeiro, Brazil. Memórias do Instituto Oswaldo Cruz, 93: 425-432.

LINDSEY, J. R., 1965. Identification of Canine MicrofiIariae. Journal of the American Veterinary Medical Association, 146:1106-1113.

LUDLAM, K. W.; JACHOWSKI, L. A. \& OTTO, G. F., 1970. Potential vectors of Dirofilaria immitis. Journal of the American Veterinary Medical Association, 157:1354-1359.

MURO, A.; GENCHI, C.; CORDERO, M. \& SIMON, F., 1999. Human dirofilariasis in the European Union. Parasitology Today, 15:386-389.

NEWTON, W. L. \& WRIGHT, W. H., 1956. The occurrence of a dog filariid other than Dirofilaria immitis in the United States. Journal of Parasitology, 42:246-258.

NICOLAS, L. \& SCOLES, G. A., 1997. Multiplex polymerase chain reaction for detection of Dirofilaria immitis (Filariidea: Onchocercidae) and Wuchereria bancrofti (Filaroidea: Dipetalonematidae) in their common vector Aedes polynesiensis (Diptera: Culicidae). Journal of Medical Entomology, 34:741-744.

OTTO, G. F., 1978. The significance of microfilaremia in the diagnosis of heartworm infection. Proceedings of the Heartworm Symposium, 77:22-30.

PARKER, B. M., 1986. Presumed Dirofilaria immitis infections from field-collected mosquitoes in North Carolina. Journal of the American Mosquito Control Association, 2:231-233.

PARKER, B. M., 1993. Variation in mosquito (Diptera: Culicidae) relative abundance and Dirofilaria immitis (Nematoda: Filarioidea) vector potencial in coastal North Carolina. Journal of Medical Entomology, 30:436-442.
PATTON, S. \& FAU LKNER, C. T., 1992. Prevalence of Dirofilaria immitis and Dipetal onema reconditum infection in dogs: 805 cases (1980-1989). Journal of the American Veterinary Medical Association, 200:1533-1534.

PEREZ-SANCHEZ, R.; GOMEZ-BAUTISTA， M. \& GRANDES, A. E., 1989. Canine filariasis in Salamanca (Northwest Spain). Annals of Tropical Medicine and Parasitology, 83:143-150.

RAMALHO, G. B.; BITTENCOURT, L. S. \& BITTENCOURT, R. D. L., 1985. Il ha de Santa Rita-AL. Oito Vertentes e Dois Momentos de Síntese da Arquitetura Brasileira. Maceió: Prefeitura Municipal de Maceió.

RODRIGUES-SILVA, R.; MOURA, H.; DREYER, G. \& REY, L., 1995. Human pulmonary dirofilariasis: A review. Revista do Instituto de Medicina Tropical, 35:523-530.

SAUERMAN, D. M. \& NAYAR, J. K., 1983. A survey for natural potencial vectors of Dirofilaria immitis in Vero Beach, Florida. Mosquito News, 43:222-225.

VILLAVASO, E. J. \& STEELMAN, C. D., 1970. Laboratory and field studies of the southern house mosquito, Culex quinquefasciatus Say, Dirofilaria immitis (Leidy), in Louisiana. Journal of Medical Entomology, 7:471-476.

WALLENSTEIN, W. L. \& TIBOLA, B. J., 1960. Survey of canine filariasis in a Maryland area - Incidence of Dirofilaria immitis and Dipetal onema. Journal of the American Veterinary Medicine Association, 137:712-716.

WHO EXPERT COM MITTEE ON RABIES, 1994. Eighth Report. WHO Technical Report Series 824. Geneva: World Health Organization. 